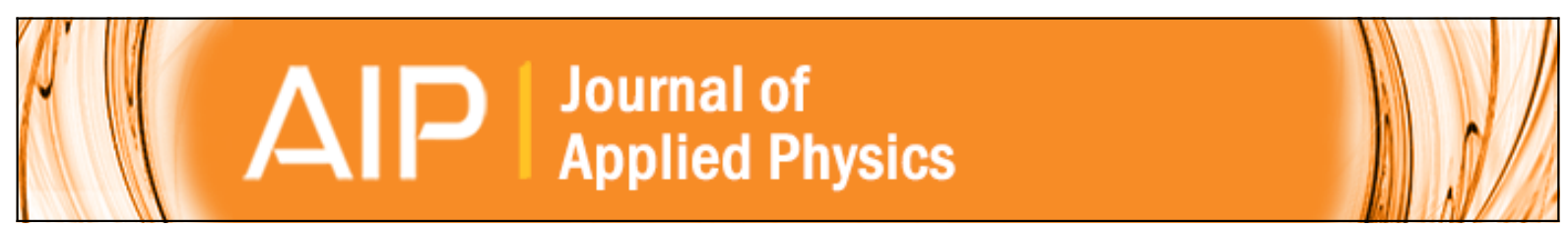

\title{
Optical resonance of a two-level atomic system
}

Munir H. Nayfeh and Ali H. Nayfeh

Citation: Journal of Applied Physics 47, 2528 (1976); doi: 10.1063/1.322969

View online: http://dx.doi.org/10.1063/1.322969

View Table of Contents: http://scitation.aip.org/content/aip/journal/jap/47/6?ver=pdfcov

Published by the AIP Publishing

\section{Articles you may be interested in}

Evidence for hydrogen two-level systems in atomic layer deposition oxides

Appl. Phys. Lett. 103, 162601 (2013); 10.1063/1.4826253

Resonance fluorescence of a two-level atom near a rough metal surface

J. Chem. Phys. 85, 567 (1986); 10.1063/1.451581

On the spectrum of a two-level system

J. Math. Phys. 26, 2792 (1985); 10.1063/1.526703

Resonance fluorescence of a two-level atom near a metal surface

J. Chem. Phys. 80, 893 (1984); 10.1063/1.446747

Two-Level Approximation in Atomic Systems

Am. J. Phys. 42, 992 (1974); 10.1119/1.1987910

MIT LINCOLN

LABORATORY CAREERS

Discover the satisfaction of innovation and service

to the nation
- Space Control

- Air \& Missile Defense

- Communications Systems \& Cyber Security

- Intelligence, Surveillance and Reconnaissance Systems

- Advanced
Electronics
- Tactical Systems
- Homeland
Protection
- Air Traffic Control

- Air Traffic Control

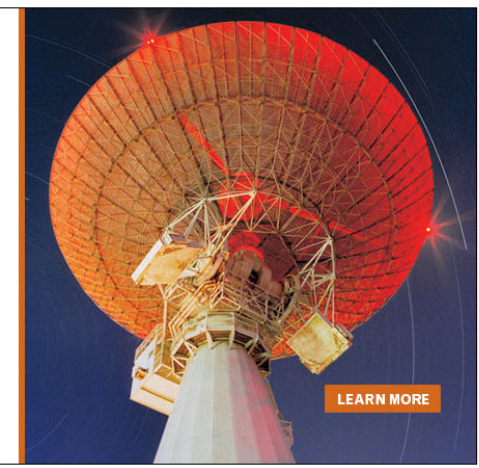




\title{
Optical resonance of a two-level atomic system
}

\author{
Munir H. Nayfeh* \\ Oak Ridge National Laboratory, Oak Ridge, Tennessee 37830
}

\begin{abstract}
Ali $H$. Nayfeh
Virginia Polytechnic Institute and State University, Blacksburg, Virginia 24061

(Received 29 September 1975; in final form 20 February 1976)

The method of multiple scales is used to derive a solution of the damped optical Bloch equations of a twolevel atomic system due to a strong pulsed field. The time dependence of the oscillations of the atomic inversion influenced by detuning and power broadening is found. The population inversion consists, in general, of three terms: a quasisteady term, a quasisteady term that decays with time, and an oscillatory term that also decays with time. In the limit of constant fields, the solution of Torrey for damped systems and that of Rabi for undamped systems are recovered. For an adiabatic switching of the field, the solution for undamped systems reduces to that of Crisp in the adiabatic following limit. An equation describing the field envelope is derived for an arbitrary amount of detuning. At exact resonance, this equation reduces to a pendulum equation, in agreement with previous analyses.
\end{abstract}

PACS numbers: $32.10 . \mathrm{Ks}$

\section{INTRODUCTION}

The damped optical Bloch equations have been used to express the response of near-resonant atoms to an applied field. Some of these near-resonant optical phenomena have strong analogues in nuclear and electron spin resonances such as optical nutation, photon echoes, and free induction decay. However, many other effects, particularly those involved in the propagation of intense pulses of radiation, are peculiar to the optical region of the frequency spectrum. This work is concerned with some nonlinear effects due to an off-resonant pulse which varies slowly compared with the precession period of the Bloch vector while its duration is not short compared with the relaxation times.

The semiclassical optical Bloch equations arise directly and naturally from the Heisenberg picture. In the rotating wave approximation and in a rotating frame, the equations describing the nearly stationary Bloch vector $\rho$ with the components $u, v$, and $w$ are $\mathrm{e}^{1}$

$$
\begin{aligned}
& \frac{d u}{d t}=-\Delta v, \\
& \frac{d v}{d t}=\Delta u+\kappa \varepsilon w, \\
& \frac{d w}{d t}=-\kappa \varepsilon v,
\end{aligned}
$$

where $\Delta=\omega-\omega_{0}$ is the detuning, $\omega_{0}$ is the frequency associated with the level in question, $\varepsilon(t, z)$ is the amplitude of the electric field $E$ defined by $E=\mathcal{E}(t, z)$ $\times \cos (\omega t-K z), u$ and $v$ are the dipole components, $w$ is the inversion, and $\kappa$ is the dipole moment.

Incoherent, possibly nonelectromagnetic, interactions are introduced into the two-level atomic dynamics by way of phenomenological relaxation constants, in the manner in which Bloch incorporated relaxation effects into the magnetic resonance theory. These constants are intended to account for the effects of collisions, of natural spontaneous decay, and of all other incoherent line-broadening processes. Thus, the damped Bloch equations are $\mathrm{e}^{\mathbf{1}}$

$$
\frac{d u}{d t}=-\gamma_{2} u-\Delta v
$$

$$
\begin{aligned}
& \frac{d v}{d t}=-\gamma_{2} v+\Delta_{u}+\kappa \varepsilon w, \\
& \frac{d w}{d t}=-\gamma_{1}\left(w-w_{e}\right)-\kappa \varepsilon v,
\end{aligned}
$$

where $\gamma_{1}=1 / T_{1}$ and $\gamma_{2}=1 / T_{2}$ are decay constants, and $w_{e}$ is the equilibrium value of the inversion when $E=0$.

Along with Eqs. (4)-(6), we introduce the classical in-phase and in-quadrature Maxwell's equations

$$
\left(K^{2}-k^{2}\right) \mathcal{E}(t, z)=2 \pi k^{2} R \kappa \int_{-\infty}^{\infty} u\left(t, z ; \Delta^{\prime}\right) g\left(\Delta^{\prime}-\Delta\right) d \Delta^{\prime},
$$

$$
\begin{gathered}
\left(K \frac{\partial}{\partial z}+k \frac{\partial}{\partial c t}\right) \varepsilon(t, z)=\pi k^{2} R \kappa \int_{-\infty}^{\infty} v\left(t, z ; \Delta^{\prime}\right) g\left(\Delta^{\prime}-\Delta\right) d \Delta^{\prime}, \\
g\left(\Delta^{\prime}-\Delta\right)=\frac{\omega_{I}}{\pi} \frac{1}{\left(\Delta^{\prime}-\Delta\right)^{2}+\omega_{I}^{2}},
\end{gathered}
$$

where $R$ is the atomic density, $K$ is the wave number, $k=\omega / c$ is the vacuum wave number, $g\left(\Delta^{\prime}-\Delta\right)$ is an inhomogeneous line-shape detuning function defined as the fraction of dipoles within the detuning interval $d \Delta^{\prime}$, and $\omega_{I}$ is the inhomogeneous half-width at half-maximum of $g\left(\Delta^{\prime}-\Delta\right)$.

For the case of continuous fields (i.e., $\mathcal{E}$ is a constant), $\mathrm{Rabi}^{2}$ and Torrey ${ }^{3}$ gave detailed exact solutions for the undamped and damped Bloch equations, respectively. Under certain conditions, it is possible to remove the restriction that $\varepsilon$ be constant and find approximate solutions to the damped Bloch equations. Crisp ${ }^{4}$ presented an approximate solution by utilizing the adiabatic following limit, ${ }^{5}$ in which $\varepsilon(t, z)$ and the Bloch vector components vary slowly on the scale $\Delta^{-1}$.

In this paper, we use the condition that the Bloch vector components are slowly varying functions of time and present an approximate solution of the damped Bloch vector equations by using the method of multiple scales. ${ }^{6}$ This technique has been successfully used by the author $s^{7}$ to solve the density-matrix equations under the influence of a strong pulsed field. We consider square as well as adiabatic pulses so that the results for a constant field can be easily recovered and the 
oscillations due to initial polarizations of the system can be derived.

\section{AN APPROXIMATE SOLUTION}

We assume that the field amplitude $\varepsilon(t, z)$ varies slowly with respect to the time scale $\Omega^{-1}$, where $\Omega$ is the the precession frequency of the Bloch vector equations, and express this fact by writing $\mathcal{E}=\mathcal{E}(\tau, z)$, where $\tau=\epsilon t$ with $\epsilon$ being the small parameter. Since Eqs. (4) $-(6)$ are a system of equations having slowly varying coefficients, one can use the method of multiple scales ${ }^{6}$ to determine an approximate solution to these equations. To this end, we let

$$
\begin{aligned}
& u=F_{1}(\tau ; \epsilon) \exp (\eta)+G_{1}(\tau ; \epsilon), \\
& v=F_{2}(\tau ; \epsilon) \exp (\eta)+G_{2}(\tau ; \epsilon), \\
& w=F_{3}(\tau ; \epsilon) \exp (\eta)+G_{3}(\tau ; \epsilon),
\end{aligned}
$$

where

$$
\frac{d}{d t}=\xi(\tau)
$$

Substituting Eqs. (10)-(13) into Eqs. (4)-(6) and equating the coefficients of $\exp (n \eta)$ on both sides with $n=0$ and 1 , we obtain

$$
\begin{aligned}
& \epsilon G_{1}^{\prime}+\gamma_{2} G_{1}+\Delta G_{2}=0, \\
& \epsilon G_{2}^{\prime}+\gamma_{2} G_{2}-\Delta G_{1}-\kappa \mathcal{E} G_{3}=0, \\
& \epsilon G_{3}^{\prime}+\gamma_{1} G_{3}+\kappa \mathcal{E} G_{2}=\gamma_{1} w_{e}, \\
& \epsilon F_{1}^{\prime}+\left(\xi+\gamma_{2}\right) F_{1}+\Delta F_{2}=0, \\
& \epsilon F_{2}^{\prime}+\left(\xi+\gamma_{2}\right) F_{2}-\Delta F_{1}-\kappa \mathcal{E} F_{3}=0, \\
& \epsilon F_{3}^{\prime}+\left(\xi+\gamma_{1}\right) F_{3}+\kappa \mathcal{E} F_{2}=0,
\end{aligned}
$$

where primes denote differentiation with respect to the argument.

To solve these equations, we expand the $G$ 's and $F$ 's in power series in terms of $\epsilon$ as

$$
\begin{aligned}
& G_{n}(\tau ; \epsilon)=G_{n 0}(\tau)+\epsilon G_{n 1}(\tau)+\cdots \circ, \\
& F_{n}(\tau ; \epsilon)=F_{n 0}(\tau)+\epsilon F_{n 1}(\tau)+\cdots \circ,
\end{aligned}
$$

for $n=1,2$, and 3 .

Substituting Eq. (20) into Eqs. (14)-(16) and equating the coefficients of $\epsilon^{0}$, we have

$$
\begin{aligned}
& \gamma_{2} G_{10}+\Delta G_{20}=0, \\
& \gamma_{2} G_{20}-\Delta G_{10}-\kappa \varepsilon G_{30}=0, \\
& \gamma_{1} G_{30}+\kappa \varepsilon G_{20}=\gamma_{1} w_{e} .
\end{aligned}
$$

The solutions of these equations are

$$
\begin{aligned}
& G_{10}=-\kappa \Delta \gamma_{1} \Gamma \mathcal{E} w_{e}, \\
& G_{20}=\kappa \gamma_{1} \gamma_{2} \Gamma \varepsilon w_{e}, \\
& G_{30}=\gamma_{1}\left(\Delta^{2}+\gamma_{2}^{2}\right) \Gamma w_{e},
\end{aligned}
$$

where

$$
\Gamma=\left[\gamma_{1} \Delta^{2}+\gamma_{2}\left(\gamma_{1} \gamma_{2}+\kappa^{2} \mathcal{E}^{2}\right)\right]^{-1} .
$$

To determine the $F_{n m}$, we substitute Eq. (21) into Eqs. (17)-(19), equate the coefficients of $\epsilon^{\circ}$ and $\epsilon$ on both sides, and obtain order $\epsilon^{0}$

$$
\begin{aligned}
& \left(\xi+\gamma_{2}\right) F_{10}+\Delta F_{20}=0, \\
& \left(\xi+\gamma_{2}\right) F_{20}-\Delta F_{10}-\kappa \varepsilon F_{30}=0, \\
& \left(\xi+\gamma_{1}\right) F_{30}+\kappa \varepsilon F_{20}=0 ;
\end{aligned}
$$

order $\epsilon$

$$
\begin{aligned}
& \left(\xi+\gamma_{2}\right) F_{11}+\Delta F_{21}=-F_{10}^{\prime}, \\
& \left(\xi+\gamma_{2}\right) F_{21}-\Delta F_{11}-\kappa \varepsilon F_{31}=-F_{20}^{\prime}, \\
& \left(\xi+\gamma_{1}\right) F_{31}+\kappa \varepsilon F_{21}=-F_{30}^{\prime} .
\end{aligned}
$$

As will be evident later, we need both the equations of $O\left(\epsilon^{0}\right)$ as well as $O(\epsilon)$ to obtain a solution that is valid to $O\left(\epsilon^{0}\right)$.

The homogeneous equations $(29)-(31)$ have a nontrivial solution if, and only if, the determinant of their coefficient matrix vanishes. This condition leads to the following eigenvalue equation for $\xi$ :

$$
\left(\xi+\gamma_{1}\right)\left(\xi+\gamma_{2}\right)^{2}+\kappa^{2}\left(\xi+\gamma_{2}\right) \mathcal{E}^{2}+\Delta^{2}\left(\xi+\gamma_{1}\right)=0
$$

Since Eq. (35) is cubic, it has three roots. In general, one of them is real and the other two are complex conjugates of each other; that is,

$$
\xi_{1}=a, \quad \xi_{2}=b+i \Omega, \quad \xi_{3}=b-i \Omega,
$$

where $a, b$, and $\Omega$ are real functions of $\tau$. Corresponding to each of these eigenvalues, Eqs. (29)-(31) yield the following eigenvectors:

$$
\begin{aligned}
& F_{10}=A_{m}(\tau), \\
& F_{20}=-\frac{\xi_{m}+\gamma_{2}}{\Delta} A_{m}(\tau), \\
& F_{30}=\frac{\kappa \mathcal{E}\left(\xi_{m}+\gamma_{2}\right)}{\Delta\left(\xi_{m}+\gamma_{1}\right)} A_{m}(\tau),
\end{aligned}
$$

for $m=1,2$, and 3 . The functions $A_{m}$ are still undetermined at this level of approximation; they are determined by invoking the solvability condition at the next level of approximation. This is the reason for writing down the terms of $O(\epsilon)$ as well as those of $O\left(\epsilon^{0}\right)$ in Eqs. (17)-(19).

Since the determinant of the coefficient matrix of the homogeneous parts of Eqs. (32)-(34) vanishes according to Eq. (35), if one attempts to solve these equations by using Cramer's rule he will find that $F_{11}, F_{21}$, and $F_{31}$ are unbounded, and hence Eqs. (32)-(34) are unsolvable unless all the numerators vanish. The vanishing of these numerators yields the following solvability condition:

$$
\left|\begin{array}{ccc}
F_{10}^{\prime} & \Delta & 0 \\
F_{20}^{\prime} & \xi+\gamma_{2} & -\kappa \mathcal{E} \\
F_{30}^{\prime} & \kappa \mathcal{E} & \xi+\gamma_{1}
\end{array}\right|=0,
$$

or

$\left[\left(\xi+\gamma_{1}\right)\left(\xi+\gamma_{2}\right)+\kappa^{2} \mathcal{E}^{2}\right] F_{10}^{\prime}-\Delta\left(\xi+\gamma_{1}\right) F_{20}^{\prime}-\Delta \kappa \varepsilon F_{30}^{\prime}=0$.

Substituting for the $F_{m 0}$ from Eqs. (37)-(39) into Eq. (41), using Eq. (35), and integrating the resulting equation, we obtain 


$$
\begin{aligned}
A_{m} & =c_{m} \Lambda\left(\xi_{m}\right) \\
& \equiv c_{m} \Delta\left[\Delta^{2}-\left(\xi_{m}+\gamma_{2}\right)^{2}+\kappa^{2}\left(\xi_{m}+\gamma_{2}\right)^{2}\left(\xi_{m}+\gamma_{1}\right)^{-2} \varepsilon^{2}\right]^{-1 / 2},
\end{aligned}
$$

where the $c_{m}$ are complex constants in general which can be deter mined from the initial conditions.

With the above results, we find the following firstorder uniform expansion:

$$
\begin{aligned}
u= & \hat{c}_{1} \Delta \Lambda(a) \exp \left[\int a(\tau) d t\right]+\hat{c}_{2} \chi(\tau) \exp \left[\int b(\tau) d t\right] \\
& \times \cos \left[\int \Omega(\tau) d t+\theta(\tau)+\beta\right]-\kappa \Delta \gamma_{1} \Gamma(\tau) \mathcal{E}(\tau) w_{e}+O(\epsilon),(43) \\
v= & -\left[\hat{c}_{1}\left(a+\gamma_{2}\right) \Lambda(a) \exp \left(\int a d t\right)+\hat{c}_{2} \chi \chi_{1} \exp \left(\int b d t\right)\right. \\
& \left.\times \cos \left(\int \Omega d t+\theta+\theta_{1}+\beta\right)\right]+\kappa \gamma_{1} \gamma_{2} \Gamma \varepsilon w_{e}+O(\epsilon) \\
w= & \kappa \varepsilon\left[\hat{c}_{1} \frac{a+\gamma_{2}}{a+\gamma_{1}} \Lambda(a) \exp \left(\int a d t\right)+\hat{c}_{2} \chi \chi_{2} \exp \left(\int b d t\right)\right. \\
& \left.\times \cos \left(\int \Omega d t+\theta+\theta_{2}+\beta\right)\right]+\gamma_{1}\left(\Delta^{2}+\gamma_{2}^{2}\right) \Gamma w_{e}+O(\epsilon),
\end{aligned}
$$

where $\hat{c}_{1}, \hat{c}_{2}$, and $\beta$ are real constants to be determined from the initial conditions

$$
\begin{aligned}
& \chi(\tau) \exp [i \theta(\tau)]=\Lambda[b(\tau)+i \Omega(\tau)] \\
& \chi_{1}(\tau) \exp \left[i \theta_{1}(\tau)\right]= b(\tau)+\gamma_{2}+i \Omega(\tau) \\
& \chi_{2}(\tau) \exp \left[i \theta_{2}(\tau)\right]= {\left[b(\tau)+\gamma_{2}+i \Omega(\tau)\right] } \\
& \times\left[b(\tau)+\gamma_{1}+i \Omega(\tau)\right]^{-1} .
\end{aligned}
$$

The functions $a(\tau), b(\tau)+i \Omega(\tau)$, and $b(\tau)-i \Omega(\tau)$ are the three roots of the cubic algebraic Eq. (35). For general values of $\gamma_{1}$ and $\gamma_{2}$, and for a given value of $\varepsilon(\tau)$, one can solve the cubic equation (35) to obtain $a, b$, and $\Omega$, and hence $u, v$, and $w$.

When $\varepsilon(t, z)$ is a constant function of time, the present solution has the same for $m$ as that obtained by Torrey using Laplace transform techniques.

For special cases such as equal decay constants or no damping, the roots of Eq. (35) and hence Eqs. (43)(45) may have simple forms as demonstrated below.

\section{THE CASE OF EQUAL DECAY CONSTANTS}

When $\gamma_{1}=\gamma_{2}=\gamma$ (which is a reasonable assumption for strong collisions),

$$
\text { and } \begin{aligned}
\xi_{1} & =a=-\gamma \\
\xi_{2} & =b+i \Omega=-\gamma+i\left[\Delta^{2}+\kappa^{2} \varepsilon^{2}(\tau)\right]^{1 / 2} .
\end{aligned}
$$

In deriving Eqs. (39) and (42), we assumed that $\xi_{m}$ $+\gamma_{1} \neq 0$. However, when $\gamma_{1}=\gamma_{2}, \xi_{1}+\gamma=0$. In this case, we set $\xi_{1}+\gamma=0$ in Eqs. (29)-(31) and (41); the result is

$$
\begin{aligned}
& F_{10}=A_{1}(\tau), \quad F_{20}=0, \quad F_{30}=-[\Delta / \kappa \mathcal{E}(\tau)] A_{1}(\tau), \\
& \kappa \mathcal{E} F_{10}^{\prime}-\Delta F_{30}^{\prime}=0 .
\end{aligned}
$$

Combining these equations and solving for $A_{1}(\tau)$, we have

$$
A_{1}(\tau)=c_{1} \kappa \varepsilon / \Omega \text {. }
$$

Therefore, Eqs. (43) $-(45)$ reduce to

$$
\begin{aligned}
u= & \frac{c_{1} \kappa \mathcal{E}(\tau)}{\Omega(\tau)} \exp (-\gamma t)+\frac{\tilde{c}_{2} \Delta}{\Omega(\tau)} \exp (-\gamma t) \cos \left[\int \Omega(\tau) d t+\beta\right] \\
& -\kappa \mathcal{E}(\tau) w_{e}\left(\Omega^{2}+\gamma^{2}\right)^{-1}+O(\epsilon),
\end{aligned}
$$

$$
\begin{aligned}
v= & \tilde{c}_{2} \exp (-\gamma t) \sin \left(\int \Omega d t+\beta\right) \\
& +\kappa \varepsilon \gamma w_{e}\left(\Omega^{2}+\gamma^{2}\right)^{-1}+O(\epsilon), \\
w= & -\frac{c_{1} \Delta}{\Omega}+\frac{\tilde{c}_{2} \kappa \varepsilon}{\Omega} \exp (-\gamma t) \cos \left(\int \Omega d t+\beta\right) \\
& +\left(\Delta^{2}+\gamma^{2}\right) w_{e}\left(\Omega^{2}+\gamma^{2}\right)^{-1}+O(\epsilon) .
\end{aligned}
$$

\section{UNDAMPED CASE}

In the absence of damping, $\gamma_{1}=\gamma_{2}=0$,

$$
\xi_{1}=a=0
$$

and

$$
\xi_{2}=b+i \Omega=i\left[\Delta^{2}+\kappa^{2} \mathcal{E}^{2}(\tau)\right]^{1 / 2} .
$$

In this case, we note that

$$
\lim _{\substack{\gamma_{1} \rightarrow 0 \\ \gamma_{2} \rightarrow 0}} \gamma_{1} \Gamma=0 .
$$

Hence, Eqs. $(43)-(45)$ reduce to

$$
\begin{aligned}
& u=\frac{c_{1} \kappa \varepsilon(\tau)}{\Omega(\tau)}+\frac{\tilde{c}_{2} \Delta}{\Omega(\tau)} \cos \left(\int \Omega(\tau) d t+\beta\right)+O(\epsilon), \\
& v=\tilde{c}_{2} \sin \left(\int \Omega d t+\beta\right)+O(\epsilon), \\
& w=-\frac{c_{1} \Delta}{\Omega}+\frac{\tilde{c}_{2} \kappa \varepsilon}{\Omega} \cos \left(\int \Omega d t+\beta\right)+O(\epsilon) .
\end{aligned}
$$

For the case of a rapidly switched field [i.e. , $\varepsilon(-\infty, z ; \Delta) \neq 0]$ and for the general initial conditions

$u=u_{0}, \quad v=v_{0}$, and $w=w_{0}$ at $z=-\infty$,

Eqs. (55) $-(57)$ become

$$
\begin{aligned}
u= & \frac{\kappa \varepsilon(\tau)\left[u_{0} \kappa \varepsilon(-\infty)-w_{0} \Delta\right]}{\Omega(\tau) \Omega(-\infty)}+\frac{\Delta}{\Omega(\tau) \Omega(-\infty)} \\
& \times\left[\left[u_{0} \Delta+w_{0} \kappa \varepsilon(-\infty)\right] \cos \left(\int_{-\infty}^{t} \Omega(\tau) d t\right)\right. \\
& \left.-v_{0} \sin \left(\int_{-\infty}^{t} \Omega(\tau) d t\right)\right]+O(\epsilon)
\end{aligned}
$$

$$
\begin{aligned}
v= & v_{0} \cos \left(\int_{-\infty}^{t} \Omega d t\right)+\frac{1}{\Omega(-\infty)}\left[u_{0} \Delta+w_{0} \kappa \varepsilon(-\infty)\right] \\
& \times \sin \left(\int_{-\infty}^{t} \Omega d t\right)+O(\epsilon),
\end{aligned}
$$

$$
\begin{aligned}
w= & -\frac{\Delta\left[u_{0} \kappa \varepsilon(-\infty)-w_{0} \Delta\right]}{\Omega \Omega(-\infty)}+\frac{\kappa \mathcal{E}}{\Omega \Omega(-\infty)} \\
& \times\left[\left[u_{0} \Delta+w_{0} \kappa \mathcal{E}(-\infty)\right] \cos \left(\int_{-\infty}^{t} \Omega d t\right)\right. \\
& \left.-v_{0}\left(\int_{-\infty}^{t} \Omega d t\right)\right]+O(\epsilon) .
\end{aligned}
$$

When the atom is initially in the ground state, $u_{0}=v_{0}$ $=0$ and $w_{0}=-1$, and Eqs. (59)-(61) reduce to

$$
u=\frac{\kappa \Delta}{\Omega(\tau) \Omega(-\infty)}\left[\varepsilon(\tau)-\varepsilon(-\infty) \cos \left(\int_{-\infty}^{t} \Omega(\tau) d t\right)\right]+O(\epsilon),
$$

$v=-\frac{\kappa \varepsilon(-\infty)}{\Omega(-\infty)} \sin \left(\int_{-\infty}^{t} \Omega(\tau) d t\right)+O(\epsilon)$, 


$$
\begin{aligned}
w= & -\frac{1}{\Omega(\tau) \Omega(-\infty)} \\
& \times\left[\Delta^{2}+\kappa^{2} \mathcal{E}(\tau) \mathcal{E}(-\infty) \cos \left(\int_{-\infty}^{t} \Omega(\tau) d t\right)\right]+O(\epsilon) .
\end{aligned}
$$

When the field is constant, $\mathcal{E}(\tau)=\mathcal{E}(-\infty)$ and $\Omega(\tau)$ $=\Omega(-\infty)$. In this case, the lower limits in the integrals of Eqs. (62)-(64) are replaced by zero, and these equations reduce to

$$
\begin{aligned}
& u=\left(2 \kappa \varepsilon \Delta / \Omega^{2}\right) \sin ^{2} \frac{1}{2} \Omega t+O(\epsilon), \\
& v=-(\kappa \varepsilon / \Omega) \sin \Omega t+O(\epsilon), \\
& w=-1+\frac{2 \kappa^{2} \varepsilon^{2}}{\Omega^{2}} \sin ^{2} \frac{1}{2} \Omega t+O(\epsilon),
\end{aligned}
$$

which agree with those of Rabi. ${ }^{2}$

In the adiabatic following limit $\varepsilon(-\infty)=0$, and Eqs. $(62)-(64)$ reduce to

$$
\begin{aligned}
& u=\frac{\kappa \varepsilon(\tau)}{\left[\Delta^{2}+\kappa^{2} \mathcal{E}^{2}(\tau)\right]^{1 / 2}}+O(\epsilon), \\
& v=O(\epsilon), \\
& w=-\frac{\Delta}{\left[\Delta^{2}+\kappa^{2} \mathcal{E}^{2}(\tau)\right]^{1 / 2}}+O(\epsilon),
\end{aligned}
$$

which agree with the solution of $\mathrm{Crisp}^{4}$ to $O(\varepsilon)$. A higherorder correction to $v$ can be obtained from Eq. (34) with $\xi+\gamma_{1}=0$. The result is

$$
v=-\frac{\Delta \kappa \mathcal{E}^{\prime}(\tau) \epsilon}{\left[\Delta^{2}+\kappa^{2} \mathcal{E}^{2}(\tau)\right]^{3 / 2}}+O\left(\epsilon^{2}\right)
$$

It can be easily shown that this solution agrees with the exact solution for self-induced transparency in the linear regime. ${ }^{4}$

\section{EXACT RESONANT CASE}

As mentioned before, the present solution is valid when the field variation is small compared with $\Omega$. For small fields, this condition is satisfied only for large $\Delta$, while for strong fields, the solution is valid for all detunings including the zero case. Thus, for a square pulse for which $\varepsilon(-\infty)$ is not small, as $\Delta \rightarrow 0$, Eqs. (62) $-(64)$ reduce to

$$
\begin{aligned}
& u=0 \\
& v=-\sin \left[\int_{-\infty}^{t} \kappa \mathcal{E}(\tau) d t\right], \\
& w=-\cos \left[\int_{-\infty}^{t} \kappa \mathcal{E}(\tau) d t\right],
\end{aligned}
$$

which is the exact solution. Comparing Eqs. (72)-(74) for the exact resonant case with Eqs. (62)-(64) for the off-resonant case, we conclude that the off-resonant dipole response to the field cannot be written in the same form as the exact resonant response but with a detuning-dependent amplitude. In other words, the factorization assumption ${ }^{8}$ does not hold according to the present theory.

\section{PULSE ENVELOPE}

Substituting for $v$ from Eq. (63) into Eq. (8) and assuming that $\mathcal{\varepsilon}(t, z)=\mathcal{E}(\xi)$, where $\xi=t-z / V$ and $V$ is the constant pulse phase velocity, we obtain

$\ddot{\theta}=\alpha \int_{-\infty}^{\infty} \frac{\dot{\theta}(-\infty) \sin \left[\int_{-\infty}^{t}\left(\ddot{\theta}^{2}+\Delta^{\prime 2}\right)^{1 / 2} d t\right]}{\left[\ddot{\theta}^{2}(-\infty)+\Delta^{\prime^{2}}\right]^{1 / 2}} g\left(\Delta^{\prime}-\Delta\right) d \Delta^{\prime}$,

where $\dot{\theta}=\kappa \varepsilon, \alpha=\pi k^{2} R \kappa^{2}(K / V-k / c)^{-1}$, and dots denote differentiation with respect to $\xi$.

When $\Delta=0$, Eq. (75) reduces to

$$
\ddot{\theta}=\alpha \sin \theta
$$

which agrees with previous analyses. ${ }^{1}$ Equation (76) shows also that the factorization assumption ${ }^{8}$ does not hold.

\section{CONCLUDING REMARKS}

We used the method of multiple scales to obtain an approximate solution of the damped Bloch equations, for an arbitrary amount of detuning, due to the influence of a strong pulsed field. This solution can be specialized to recover the solutions of $\mathrm{Rabi}^{2}$ and Torrey ${ }^{3}$ for constant fields, and the solution of $\mathrm{Crisp}^{4}$ in the adiabatic following limit. A number of special cases are discussed that include strong collisions, exact resonance, and undamped systems. The problem of pulse envelope is also discussed.

*Post Doctoral Research appointment through the University of Kentucky and supported by the Los Alamos Scientific Laboratory.

${ }^{1} \mathrm{~L}$. Allen and J.H. Eberly, Optical-Resonance and Two-Level Atoms (Wiley-Interscience, New York, 1975), Chap. 2.

${ }^{2}$ I. I. Rabi, Phys, Rev. 51, 652 (1937).

${ }^{3}$ H. C. Torrey, Phys. Rev. 76, 1059 (1949).

${ }^{4}$ M. D. Crisp, Phys. Rev. A 8, 2128 (1973).

${ }^{5}$ D. Grischkowsky, Phys. Rev. Lett. 24, 866 (1970); D. Grischkowsky and J.A. Armstrong, Phys. Rev. A 6, 1566 (1972); D. Grischkowsky, Phys. Rev. A 7, 2096 (1973); D. Grischkowsky, E. Courtens, and J. A. Armstrong, Phys. Rev. Lett. 31, 422 (1973).

${ }^{6}$ A.H. Nayfeh, Perturbation Methods (Wiley-Interscience, New York, 1973), Chap. 6.

${ }^{7}$ M.H. Nayfeh and A. H. Nayfeh, J. Appl. Phys. 46, 4862 (1975).

${ }^{8}$ L. Matulic and J.H. Eberly, Phys. Rev. A 6, 822 (1972); 6, 1258E (1972). 